\title{
PAPR Reduction of FBMC by Clipping and Its Iterative Compensation
}

\author{
Zsolt Kollár and Péter Horváth \\ Department of Broadband Infocommunications and Electromagnetic Theory, Budapest University of Technology and Economics, \\ Budapest 1111, Hungary
}

Correspondence should be addressed to Zsolt Kollár, kollar@mht.bme.hu

Received 5 February 2012; Revised 22 April 2012; Accepted 2 May 2012

Academic Editor: Dov Wulich

Copyright (๑) 2012 Z. Kollár and P. Horváth. This is an open access article distributed under the Creative Commons Attribution License, which permits unrestricted use, distribution, and reproduction in any medium, provided the original work is properly cited.

Physical layers of communication systems using Filter Bank Multicarrier (FBMC) as a modulation scheme provide low out-ofband leakage but suffer from the large Peak-to-Average Power Ratio (PAPR) of the transmitted signal. Two special FBMC schemes are investigated in this paper: the Orthogonal Frequency Division Multiplexing (OFDM) and the Staggered Multitone (SMT). To reduce the PAPR of the signal, time domain clipping is applied in both schemes. If the clipping is not compensated, the system performance is severely affected. To avoid this degradation, an iterative noise cancelation technique, Bussgang Noise Cancelation (BNC), is applied in the receiver. It is shown that clipping can be a good means for reducing the PAPR, especially for the SMT scheme. A novel modified BNC receiver is presented for SMT. It is shown how this technique can be implemented in real-life applications where special requirements must be met regarding the spectral characteristics of the transmitted signal.

\section{Introduction}

In wireless communications the frequency spectrum is an essential resource. As the unlicensed spectrum is used by an increasing number of devices, the possibility of communication collision is increasing. To avoid this collision, two solutions are possible: extending the frequency limits higher to unused frequency bands at the upper end of the spectrum or reaggregating the densely used licensed frequency bands. Both ideas have disadvantages: the use of higher frequencies requires expensive specially designed analog devices; the reuse of the spectrum calls for complex, intelligent, and adaptive systems. In this paper the focus is on the reuse of the spectrum with multicarrier modulations tailored for spectrally efficient applications.

Future applications operating in the licensed bands, for example, cognitive radios, favor spectrally efficient FBMC schemes with low out-of-band leakage, minimizing harmful interference between devices using adjacent channels. In this paper two subclasses of FBMC are investigated, both allowing the use of a complex modulation alphabet: OFDM and SMT.
Both of these schemes provide relatively low out-of-band leakage.

Today OFDM [1] is the de-facto standard technique for high-speed wireless data transmission. Using OFDM, lowcomplexity modulation and demodulation can be performed by the "Inverse Fast Fourier Transform (IFFT)" and the Fast Fourier Transform (FFT), respectively. With Cyclic Prefix (CP), channel equalization can be efficiently implemented in the frequency domain. This scheme also has some drawbacks. As the PAPR of the transmitted signal is large, OFDM is highly sensitive to the nonlinear characteristics of the Power Amplifier (PA) and the D/A-A/D converters [2]. Signal preprocessing has to be applied to reduce the high PAPR of the OFDM signal, otherwise power amplification would not be efficient. Nevertheless, if the linear range of the PA is smaller than required, nonlinear effects also degrade the performance of the OFDM system. The nonlinearity introduces in-band and out-of-band distortion as well.

Numerous signal processing methods have been proposed to reduce the PAPR of OFDM $[3,4]$ such as, amplitude clipping [5], coding [6], interleaving [7], partial transmit 
sequence [8], selected mapping [9], tone reservation [10], tone injection [11], and active constellation extension [12]. Each has its own advantage and drawback. Clipping introduces distortion, some methods may require higher power, others cause data rate loss, and in some cases additional information must be transmitted to the receiver. Furthermore, the computation complexity varies for each technique.

Besides OFDM, another FBMC-based multicarrier family is being strongly investigated: the SMT $[13,14]$ scheme, which is also known as OFDM/Offset-QAM [15]. The SMT scheme has significantly reduced out-of-band leakage compared to OFDM. However, due to the absence of CP, it is more sensitive to effects of multipath propagation. The SMT signal suffers from large PAPR similarly to OFDM, which makes it especially vulnerable against nonlinearities present in the transceiver chain. For PAPR reduction of SMT signals, only the clipping technique can be applied as the consecutive symbols are overlapping, therefore it is not possible to treat them separately as in OFDM.

In this paper the baseband amplitude clipping method [5] is applied for reducing the PAPR of OFDM and SMT signals. Clipping is applied to the transmitted signal in order to align it to the linear range of the PA. Although the PAPR of the signal can be easily limited by clipping, nonlinear effects are introduced, degrading the system performance. Therefore clipping must be compensated in the receiver. The receiver-oriented turbo principle is a good candidate for the compensation of the negative side effect of clipping. Two different iterative techniques are described for OFDM receivers:

(i) Decision Aided Reconstruction (DAR) [16], where the receiver aims to reconstruct the peaks of the time domain signal.

(ii) Bussgang Noise Cancellation (BNC) [17], where the objective is to remove the clipping noise in the frequency domain.

Both methods were originally proposed using hard-decisionbased decoding procedures. Modified receivers using soft decisions are introduced in [18]. Using the soft information the receiver takes full advantage of the turbo principle, yielding better Bit Error Rates (BER). In this paper the focus is on the BNC algorithm using soft decision because it outperforms the DAR method [18].

This paper is organized as follows. First the system model applying clipping on coded FBMC signals is introduced. A short description is given of OFDM and SMT. The PAPRs and the spectral characteristics of the transmitted signals are compared. The mathematical description of the clipping effects is also presented. In the next section, a detailed description is given of the soft $\mathrm{BNC}$ receiver algorithm, introducing modifications to the method presented in $[18,19]$ in order to be applicable to SMT. The technique is explained both for OFDM and SMT schemes. The convergence analysis is given based on Extrinsic Information Transfer (EXIT) chart. The possibility to apply clipping on a real-life system is considered and modified transmitter architectures are also presented. In the closing section BER simulations are shown over ideal and frequency selective channels with Additive

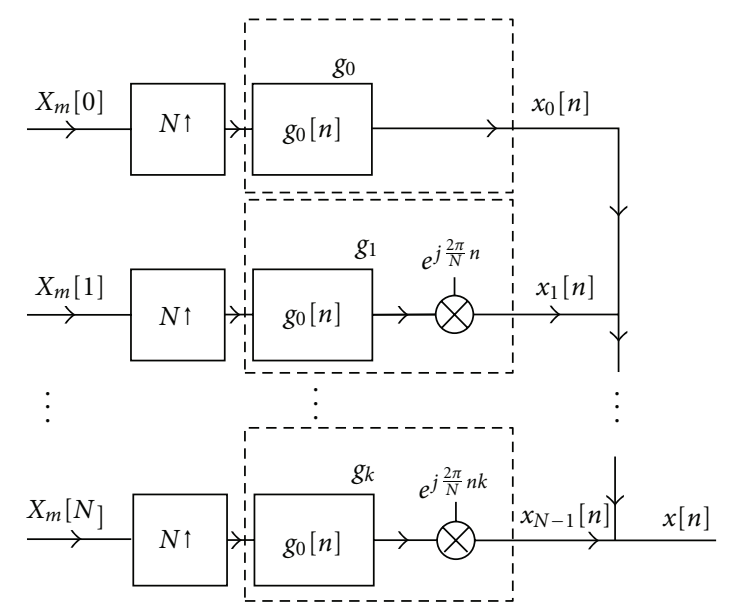

FIGURE 1: Block diagram of an FBMC modulation scheme.

White Gaussian Noise (AWGN). The paper concludes with a summary of the most important results.

\section{System Model}

2.1. FBMC Modulation Scheme. The FBMC transmit signal is constructed from $N$ parallel streams as shown in Figure 1. The input for the $m$ th symbol in the $k$ th branch $X_{k}[m]$ is selected from the complex modulation alphabet $\mathcal{A}$. The modulation symbols are upsampled by a factor of $N$ to achieve maximum data rate with critical sampling. For each upsampled signal a specially designed complex modulated prototype filter is applied having the impulse response $g_{k}[n]$ and $Z$-transform $G_{k}(z)$ as

$$
\begin{gathered}
g_{k}[n]=g_{0}[n] e^{j k(2 \pi / N)}, \quad 0<n<K N, \\
G_{k}(z)=G_{0}\left(W_{N}^{k} z\right),
\end{gathered}
$$

where $j=\sqrt{-1}$ is the imaginary unit, $W_{N}=e^{j(2 \pi / N)}$, and $K$ is the overlapping factor giving the number of overlapping impulses. The $x_{k}[n]$ output streams are summed to form the transmit signal $x[n]$ which can be expressed using (1) as

$$
x[n]=\sum_{m=-\infty}^{\infty} \sum_{k=0}^{N-1} X_{m}[k] g_{0}[n-m N] e^{j k(n-m N)(2 \pi / N)} .
$$

In the receiver a similar filter bank is used to separate the $N$ data streams. The separated streams are downsampled to retrieve the transmitted complex modulation values. The FBMC scheme can be implemented in a computationally efficient way using IFFT and a polyphase decomposition of the filters $G_{k}(z)[20]$.

2.1.1. OFDM Transmitter. The OFDM scheme is a special class of FBMC where a prototype filter with a rectangular impulse response is applied. This leads to a simplified structure where the consecutive symbols do not overlap, 


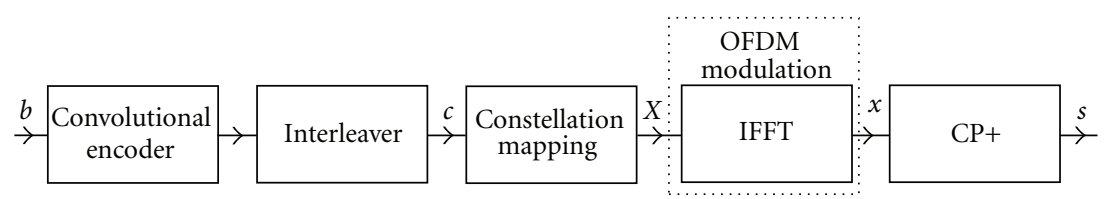

FIGURE 2: Block diagram of an OFDM transmitter.

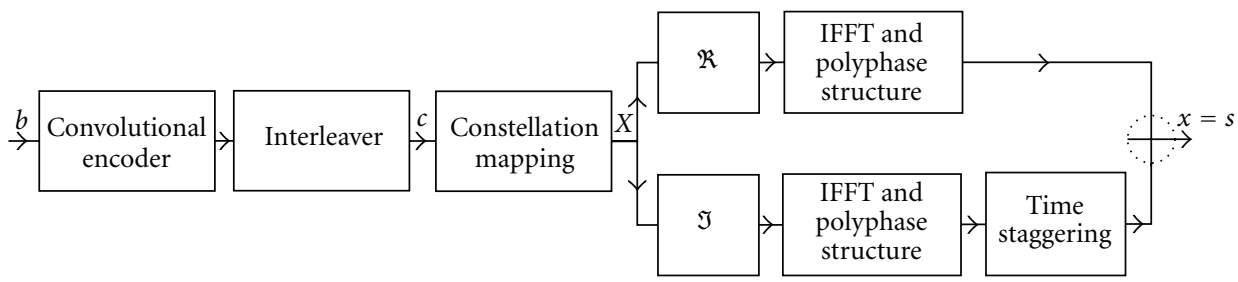

FIGURE 3: Block diagram of an SMT transmitter.

that is, $K=1$. As a result, the samples of an OFDM symbol can be expressed by simplifying (3) as described in [1]:

$$
x[n]=\sum_{k=0}^{N-1} X[k] e^{j k n(2 \pi / N)}, \quad 0 \leq n<N .
$$

The block diagram of an OFDM transmitter is shown in Figure 2. The binary information data $b$ are encoded by a rate- $R$ convolutional encoder and the encoded bits are interleaved. The interleaved bits are mapped to $X$ complex constellation symbols from the set $\mathcal{A}$. Each $a_{k} \in \mathcal{A}$ symbol maps $M$ encoded and interleaved bits. Finally IFFT is used to modulate the subcarriers. Then, prior to transmission, a CP with $P$ samples is added to each symbol to form the transmitted signal $s$.

2.1.2. SMT Transmitter. In the SMT scheme prototype filters with overlapping impulse responses fulfilling the Nyquist criterion are applied. Due to the advantageous properties of the prototype filter bank, the SMT signal will have a better Adjacent Channel Leakage Ratio (ACLR) than OFDM. With the use of offset-QAM modulation, where the real and imaginary data are transmitted with a time offset of a half symbol duration, no data rate loss will occur compared to OFDM. Prior to transmission, the symbols are overlapped such that they can be separated at the receiver. In order to maintain orthogonality of the filter bank structure, CP can not be used in SMT systems. As a result, techniques with higher complexity must be applied in comparison to OFDM in order to combat the channel-induced intersymbol interference [21, 22]. The modulated signal for the SMT scheme can be expressed as:

$$
\begin{aligned}
x[n]=\sum_{m=-\infty}^{\infty} \sum_{k=0}^{N-1}( & \theta_{k} \mathfrak{R}\left\{X_{m}[k]\right\} g_{0}[n-m N] \\
& \left.+\theta_{k+1} \mathfrak{J}\left\{X_{m}[k]\right\} g_{0}\left[n-m N-\frac{N}{2}\right]\right) \\
& \times e^{j k(n-m N)(2 \pi / N)},
\end{aligned}
$$

where

$$
\theta_{k}= \begin{cases}1, & \text { if } k \text { is even } \\ j, & \text { if } k \text { is odd }\end{cases}
$$

An efficient implementation of (5) is to use two separate polyphase filter banks where two output signals are time staggered and added. This polyphase structure of the SMT scheme can be seen in Figure 3. A major difference compared to OFDM is the no CP is applied. The SMT transmit signal is identical to the modulated signal $s[n]=x[n]$.

\subsection{Properties of The Transmitted Signal}

2.2.1. PAPR. The PAPR is one of the quantities that describes the dynamic properties of the transmitted signal $s[n]$. The PAPR is defined as:

$$
\operatorname{PAPR}(s[n])_{\mathrm{dB}}=10 \log _{10}\left(\frac{\max \left\{|s[n]|^{2}\right\}}{P_{s}}\right),
$$

where $|s[n]|$ is the amplitude and $P_{s}$ is the average power of the transmitted signal. The PAPR curves of the transmitted symbols are analyzed in Figure 4, where the Complementary Cumulative Distribution Functions (CCDFs) of the PAPR are depicted as a function of the number of subchannels $N$ with an oversampling ratio of 4 . It can be seen that as the number of subchannels increases, the probability that the amplitude exceeds a certain PAPR threshold $\left(\mathrm{PAPR}_{0}\right)$ increases for both systems.

2.2.2. Power Spectrum Density. Spectral behavior especially regarding the ACLR is also an important property of the transmitted signal. The power spectrum density function of the transmitted signal with an oversampling factor of 4 is depicted in Figure 5 as a function of the number of subchannels. In case of OFDM the length of the CP is set to $1 / 4$ symbol duration. The adjacent channel leakage is considerably lower in SMT than in OFDM. The low out-of-band radiation makes SMT a more suitable solution for cognitive 


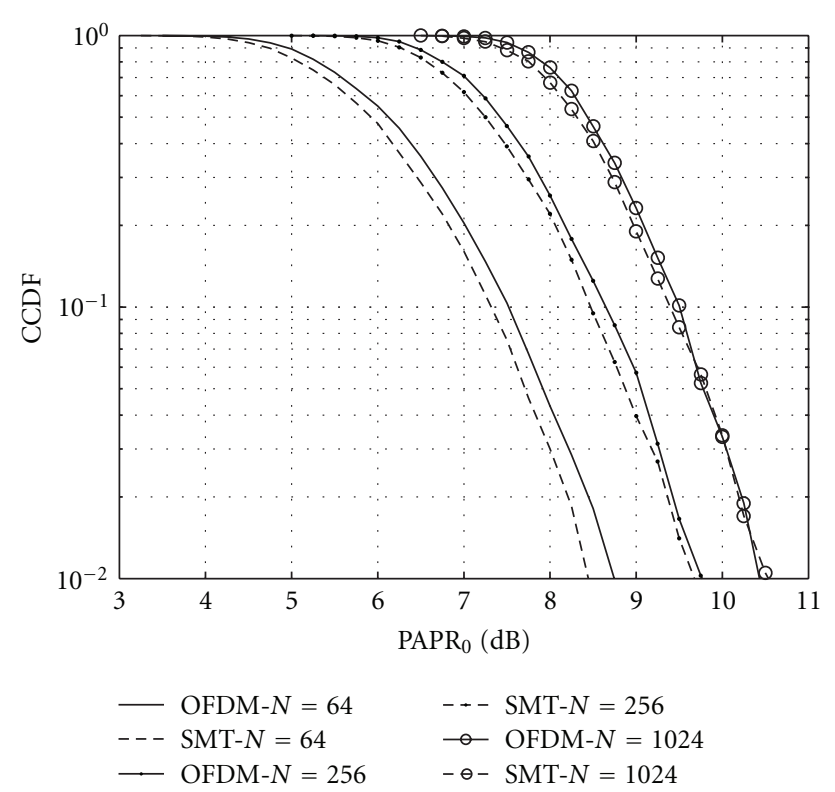

FIgURe 4: CCDF of the PAPR of the transmitted signal of OFDM and SMT for various number of subchannels. The probability that an amplitude value exceeds a certain threshold $\mathrm{PAPR}_{0}$ is visible.

radio applications where strict ACLR limits are enforced. If the number of subchannels increases, the PAPR also increases, but the spectral characteristics become more efficient, out-of-band radiation is reduced. It has been shown in [23] that in the presence of a nonlinear PA the performance of both systems degrades severely. The spectral mask is distorted, leading to a considerable amount of out-of-band leakage. A detailed analysis of the out-of-band radiation for OFDM and SMT schemes is given in [24].

2.3. Clipping. Clipping is applied to the baseband transmit signal $s[n]$ in order to reduce the PAPR. The amplitude values are limited to a threshold of $A_{\max }$. The clipped signal $s^{c}[n]$ is given as

$$
s^{c}[n]= \begin{cases}s[n] & |s[n]| \leq A_{\max } \\ A_{\max } e^{j \varphi(s[n])} & |s[n]|>A_{\max }\end{cases}
$$

where $\varphi(s[n])$ is the phase of the complex signal $s[n]$. The limiter is characterized by the clipping ratio (CR),

$$
\mathrm{CR}_{\mathrm{dB}}=20 \log _{10}(\gamma),
$$

with $\gamma=A_{\max } / \sqrt{P_{s}}$. According to Bussgang's theorem [25], the signal at the output of the limiter can be expressed as

$$
s^{c}[n]=\alpha s[n]+d[n] .
$$

The clipping noise $d[n]$ is assumed to be complex Gaussian distributed and uncorrelated with the useful signal $s[n]$ and the attenuation factor $\alpha$ is calculated as [25]

$$
\alpha=1-e^{-\gamma^{2}}+\frac{\sqrt{\pi}}{2} \gamma \operatorname{erfc}(\gamma) .
$$

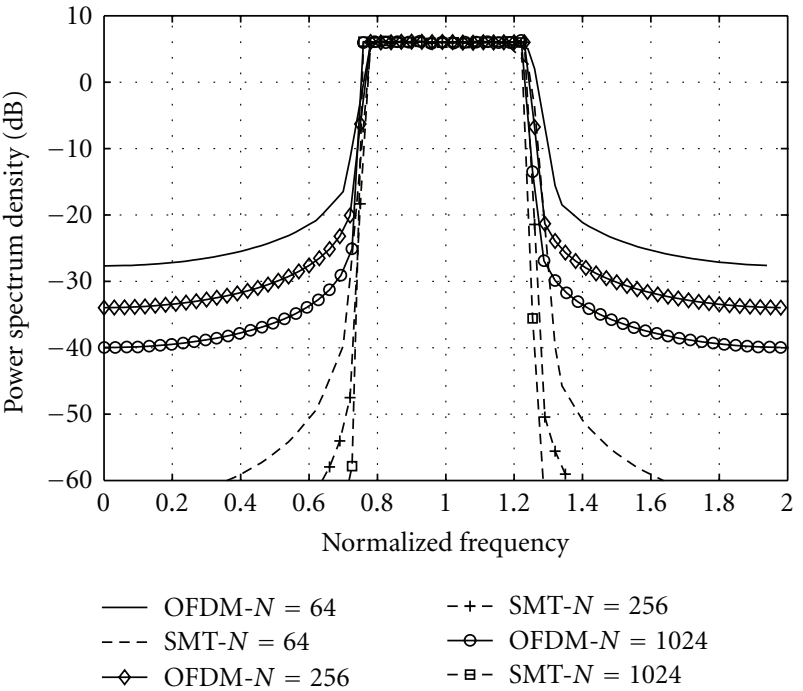

Figure 5: Power spectrum density comparison of the transmitted signals of OFDM and SMT with various number of subchannels.

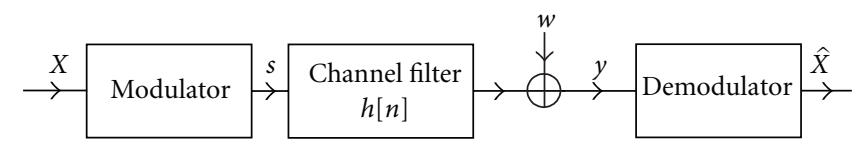

Figure 6: Block diagram of the baseband transceiver chain.

The output power of the limiter is given by

$$
P_{\text {out }}=\left(1-e^{-\gamma^{2}}\right) P_{s} .
$$

Using (10) and (12), the clipping noise power can be calculated as

$$
P_{d}=\left(1-e^{-\gamma^{2}}-\alpha^{2}\right) P_{s} .
$$

As clipping is performed on the baseband digital signal nonlinear distortions will only occur in the baseband, that is, all distortions terms will fall in-band.

2.4. Transceiver Chain. For modeling the transceiver chain the digital baseband equivalent is used. The model of the transceiver is presented in Figure 6. The radio channel is modeled as a FIR filter having a discrete impulse response $h[n]$ and a sampled AWGN term $w[n]$ with variance $\sigma_{0}^{2}=$ $N_{0} / 2$ per complex dimension. The sampled received signal can be expressed as:

$$
y[n]=s[n] * h[n]+w[n] .
$$

The receiver is assumed to have the knowledge of the channel coefficients $h[n]$.

\section{Bussgang Noise Cancellation}

3.1. BNC Turbo Detection for OFDM Systems. In OFDM systems, the $\mathrm{CP}$ of $P$ samples is assumed to be longer than 
the channel's maximum excess delay. As a result, the received symbols on the $k$ th subcarrier after OFDM demodulation can be expressed using (10) as

$$
\begin{aligned}
Y[k] & =X^{c}[k] H[k]+W[k] \\
& =\alpha X[k] H[k]+D[k] H[k]+W[k], \quad 0 \leq k<N,
\end{aligned}
$$

where $X[k], X^{c}[k], D[k], H[k]$, and $W[k]$ are the discrete Fourier transforms of the sampled signals $x[n], x^{c}[n], d[n]$, $h[n]$, and $w[n]$, respectively. The BNC receiver performs iterative equalization and detection [26]. The basic block diagram of a BNC receiver for OFDM according to [18] is shown in Figure 7. Two main subblocks are present in the figure: the BNC detector and the channel decoder. The BNC detector consists of a forward and feedback signal processing path.

3.1.1. Forward Path. The extrinsic Log-Likelihood Ratio (LLR) for each channel observation $\hat{Y}[k]$ is calculated according to [27] as

$$
L\left(b_{u, v} \mid \hat{Y}[k]\right)=\ln \frac{\sum_{a_{l} \in \mathcal{A}_{u, v}^{1}} p\left(\hat{Y}[k] \mid a_{u}=a_{l}\right)}{\sum_{a_{l} \in \mathcal{A}_{u, v}^{0}} p\left(\hat{Y}[k] \mid a_{u}=a_{l}\right)},
$$

where $\mathcal{A}_{u, v}^{1}$ and $\mathcal{A}_{u, v}^{0}$ are subsets of $\mathcal{A}_{u}$. The $v$ th bit in $a_{u}$ can be either 1 or 0 . The conditional probability density function $p\left(\hat{Y}=a_{l}\right)$ is given by [16]

$$
p(\hat{Y}[k] \mid a)=\exp \left(\frac{(\hat{Y}[k]-\alpha H[k] a)^{2}}{N_{0}+|H[k]|^{2} P_{D}^{i}}\right),
$$

where $P_{D}^{i}$ is the power of the remaining clipping noise after the $i$ th iteration. Taking into account the large number of samples and applying the central limit theorem, the clipping noise $d[n]$ can be modeled as a Gaussian distributed random variable, which is independent of the channel noise $w[n]$. Based on this assumption, passing through the linear channel filter, the power of the Bussgang noise $P_{D}$ is multiplied by $|H[k]|^{2}$. For the 0 th iteration, with no feedback, $P_{D}^{0}$ is calculated according to (13). For the next iterations, $P_{D}^{i}$ can be approximated as

$$
P_{D}^{i}=E\left\{|D[k]-\hat{D}[k]|^{2}\right\} .
$$

As the receiver does not know $D[k]$, the power of the remaining clipping noise is to be estimated as

$$
P_{D}^{i}=P_{D}^{0}-E\left\{|\hat{D}[k]|^{2}\right\}
$$

3.1.2. Feedback Path. After interleaving the extrinsic LLRs provided by the channel decoder, the soft symbols are calculated as [16]

$$
\tilde{X}_{n}=\sum_{l=0}^{2^{M}-1} a_{l} \prod_{u=0}^{M-1} P\left(b_{l, u}\right), \quad a_{l} \in \mathcal{A} .
$$

Each symbol is first weighted by the probability of the mapped bits and then summed up. Using these soft symbols a time domain estimation of the OFDM signal is performed. Clipping is applied with a level of $A_{\max }$, and the signal is converted back to the frequency domain. The attenuation factor $\alpha^{i}$ must be set in accordance with the output power of the soft mapper. If the estimated extrinsic information for the coded bits is rather low due to low channel SNR values, no clipping compensation will be performed. The clipping ratio for the ith iteration can be calculated as

$$
\gamma^{i}=\frac{A_{\max }}{\sqrt{P_{\widetilde{x}}}} .
$$

The attenuation factor for the ith iteration is calculated according to (11) using (21) as

$$
\alpha^{i}=1-e^{-\left(\gamma^{i}\right)^{2}}+\frac{\sqrt{\pi}}{2} \gamma^{i} \operatorname{erfc}\left(\gamma^{i}\right) .
$$

Following each iteration the attenuation factor in the feedback loop decreases from 1 to $\alpha$ as the estimation becomes more and more precise.

Subtracting the attenuated symbols from the clipped symbols, the estimated clipping noise can be expressed as

$$
\hat{D}[k]=\tilde{X}^{c}[k]-\alpha^{i} \tilde{X}[k], \quad 0 \leq k<N .
$$

The estimated noise term $\hat{D}$, multiplied by the channel coefficient, is then subtracted from the received symbols (15) to suppress the clipping noise

$$
\begin{aligned}
\hat{Y}[k]= & \alpha H[k] X[k]+H[k](D[k]-\hat{D}[k]) \\
& +W[k], \quad 0 \leq k<N .
\end{aligned}
$$

The 0th iteration is the case when no feedback loop is used, that is, $\hat{Y}[k]=Y[k]$. The attenuation factor $\alpha^{i}$ is monotonously decreasing, so the iteration can stop when consecutive iterations give less difference in $\alpha$ than a given limit.

The BCJR channel decoder [28] computes the extrinsic information of the deinterleaved LLRs, which are provided by the BNC detector. These extrinsic LLRs are used to suppress the clipping noise in the feedback path of the BNC detector.

3.2. Convergence Analysis. The convergence behavior of a turbo loop can be examined using the Extrinsic Information Transfer (EXIT) chart, developed by ten Brink [26]. It is used to investigate the iteration behavior of a turbo loop based on the exchange of mutual information. This powerful tool enables the tracing of mutual information exchange between the BNC detector and the channel decoder over the iterations.

The LLRs defined by (16) are modeled with an equivalent Gaussian channel [26]. The mutual information between these LLRs and the transmitted symbols $U$ which 


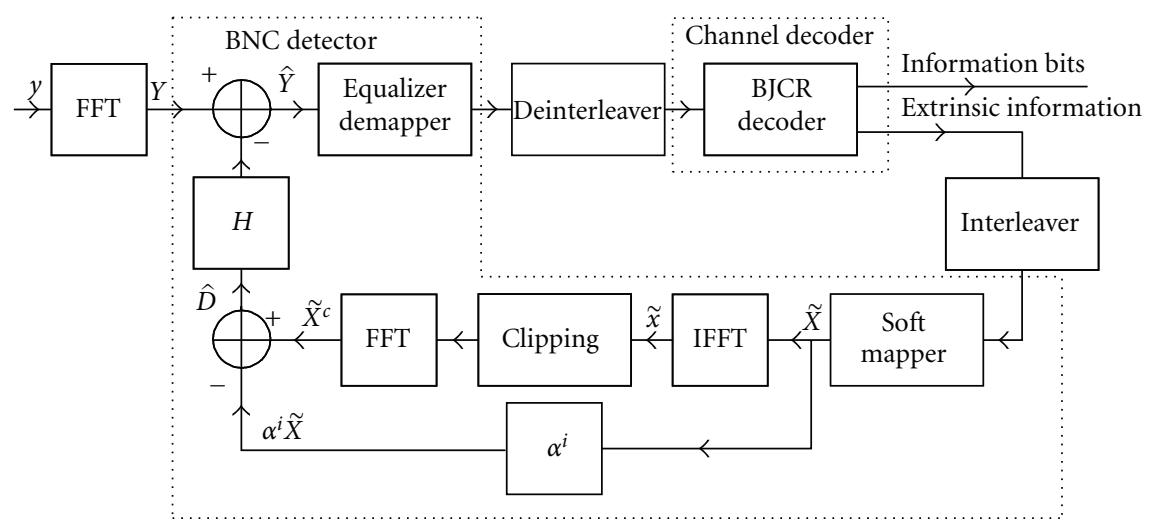

FIGURE 7: Block diagram of the Bussgang noise cancelation for OFDM.

are the realizations of $u \in\{-1,+1\}$ can be expressed with the conditional probability density function [26] as

$$
\begin{aligned}
I_{A}(U ; \mathrm{LLR})= & \frac{1}{2} \sum_{u=-1,1} \int_{-\infty}^{\infty} p_{A}(\xi \mid U=u) \\
& \cdot \log _{2} \frac{2 p_{A}(\xi \mid U=u)}{p_{A}(\xi \mid U=-1)+p_{A}(\xi \mid U=1)} \mathrm{d} \xi,
\end{aligned}
$$

where $0 \leq I_{A} \leq 1$. The binary variable $u_{k}=-1$ and $u_{k}=1$ represents the digital bits $b_{k}=0$ and $b_{k}=1$, respectively. To measure the mutual information content of the output extrinsic LLR values, the following expression is applied:

$$
\begin{aligned}
I_{E}(U ; \mathrm{LLR}) & =1-E\left\{\log _{2}\left(1+e^{-\mathrm{LLR}}\right)\right\} \\
& \approx 1-E\left\{\log _{2}\left(1+e^{-u_{k} L L R_{k}}\right)\right\} .
\end{aligned}
$$

The EXIT function of the BNC detector is not only a function of the a priori mutual information $I_{A}$ provided by the channel decoder, but it also depends on $E_{b} / N_{0}$ as $I_{E 1}=$ $f\left(I_{A 1}, E_{b} / N_{0}\right)$. The EXIT function of the channel decoder only depends on the a priori LLRs provided by the BNC detector as $I_{E 2}=f\left(I_{A 2}\right)$. The iteration steps of the turbo loop can be visualized using the EXIT functions of the BNC detector and the channel decoder. The output of the channel decoder becomes the input of the BNC detector, and the output of the detector will be the new input of the decoder in the next iteration:

$$
\begin{gathered}
I_{E 1}=f\left(I_{A 1}=I_{E 2}, \frac{E_{b}}{N_{0}}\right), \\
I_{E 2}=f\left(I_{A 2}=I_{E 1}\right) .
\end{gathered}
$$

To observe the mutual information transfer of the turbo loop, the EXIT chart is constructed from the two EXIT functions. The EXIT function of the channel decoder is plotted with swapped $x-y$ axes on top of the BNC detectors to visualize the iteration trajectory. An iteration trajectory can be seen for $E_{b} / N_{0}=4 \mathrm{~dB}$ and $E_{b} / N_{0}=12 \mathrm{~dB}$ with a channel

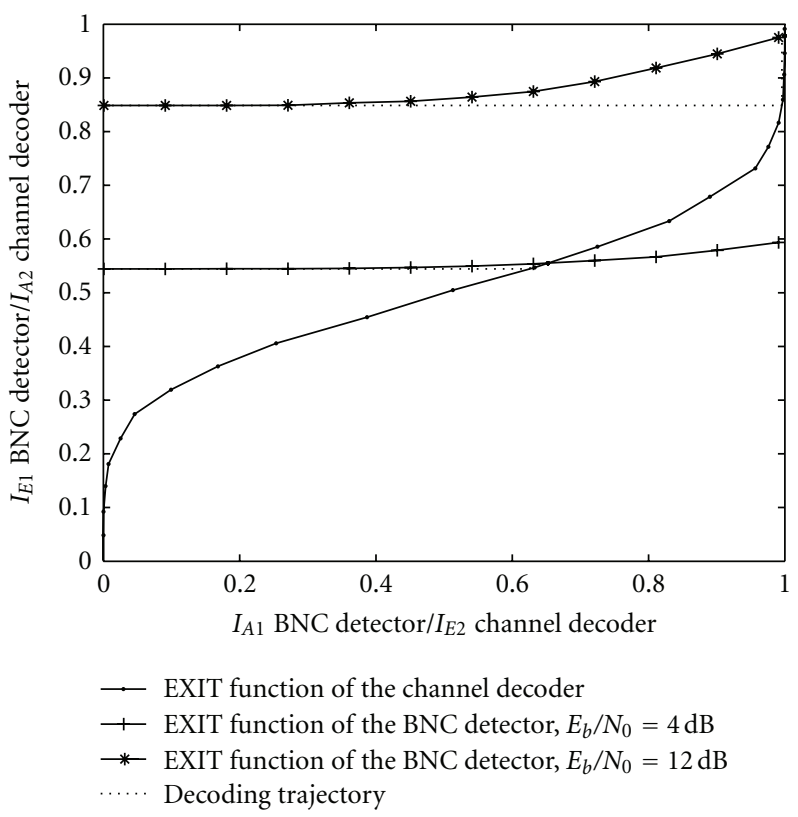

FIGURE 8: EXIT chart, with iteration trajectories of the BNC turbo receiver with an $R=1 / 2$ rate channel decoder for $E_{b} / N_{0}=4 \mathrm{~dB}$ and $E_{b} / N_{0}=12 \mathrm{~dB}$ values with $\mathrm{CR}=1 \mathrm{~dB}$

decoder rate of 12 in Figure 8. In iterative receivers the EXIT functions of both decoders have to be monotonically increasing in order to achieve convergence. It can be observed in Figure 8 that the monotony of the BNC detector is satisfactory. As the input mutual information $I_{A 1}$ increases, a growing output mutual information $I_{E 1}$ can be observed. If the extrinsic information in the feedback loop has reached the value of 1 , that is, a perfect reconstruction is achieved, meaning that the clipping noise can be fully removed and unclipped system performance can be achieved.

3.3. Modified BNC Turbo Detector for SMT Systems. For SMT scheme the blocks of the BNC receiver presented for OFDM in Figure 7 must be modified and extended. Both, the feedforward and the feedback path should be altered, due to 


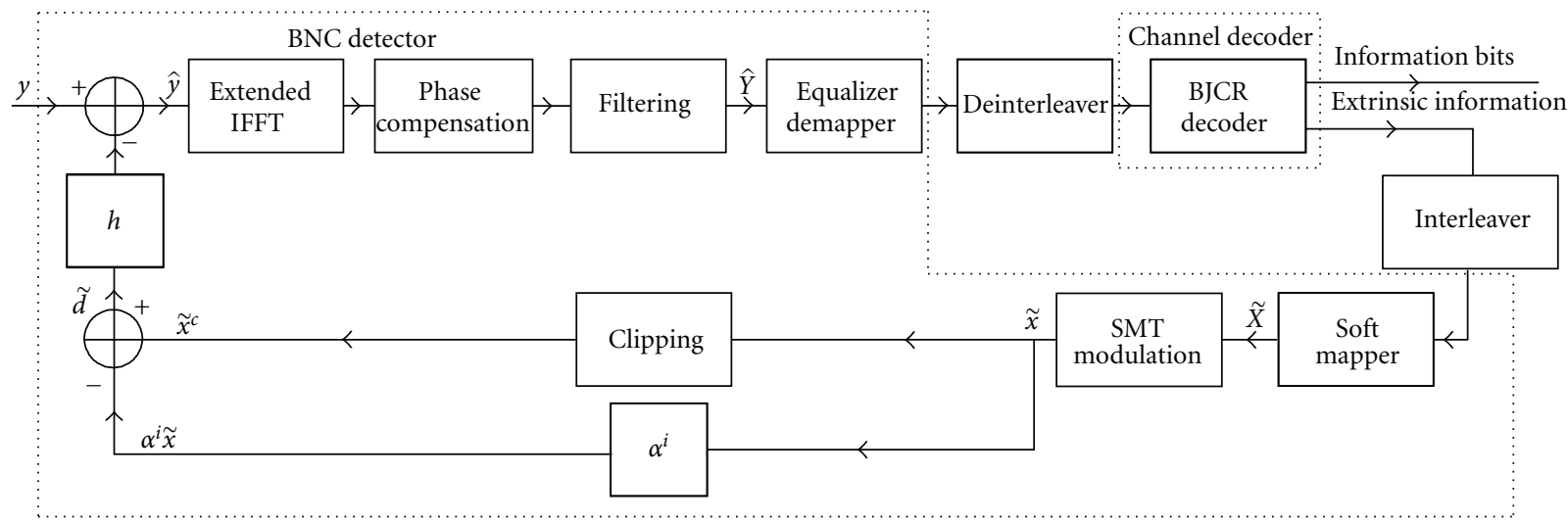

FIgURE 9: Block diagram of the modified BNC receiver for SMT.

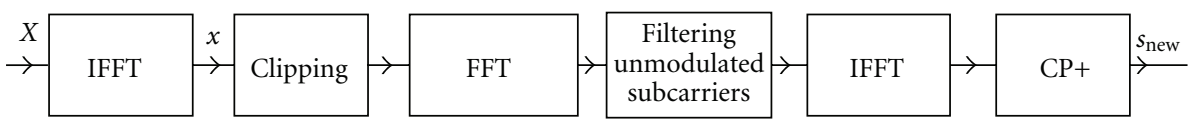

(a) OFDM

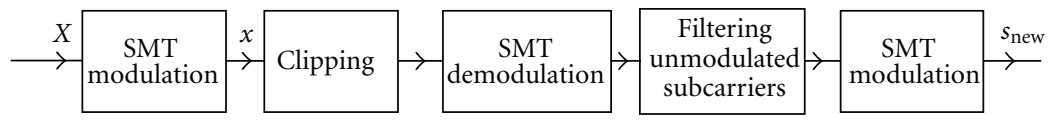

(b) SMT

FIGURE 10: Block diagram of the modified transmitter of OFDM (a) and SMT (b) systems.

the overlapping nature of the symbols and the absence of the $\mathrm{CP}$, as it can be seen in Figure 9:

(i) The compensation of the clipping noise is performed in time domain before demodulation.

(ii) In the presence of ISI only a quasi Maximum Likelihood (ML) detection of the received modulation symbols $\hat{Y}$ can be performed.

(iii) The demapping blocks have to be extended with additional signal processing blocks.

First, an enlarged FFT operation is applied [29] with a length equal to the time domain impulse duration of the prototype filter. The phase compensation of the transmission channels effect for each subcarrier in each subchannel is performed in the frequency domain. After the phase compensation, filtering is performed in the frequency domain [29]. Finally, a quasi-ML detection of the transmitted, channel distorted complex modulation values is calculated similar to the case of OFDM. Equation (17) has to be modified taking ISI into account. The probability function is approximated as

$$
p(\hat{Y}[k] \mid a) \approx \exp \left(\frac{(\hat{Y}[k]-\alpha H[k] a)^{2}}{I+N_{0}+|H[k]|^{2} P_{D}^{i}}\right)
$$

where I is the ISI term which can be calculated according to [21].

\section{Practical Application}

In real-life systems not all subcarriers are used for data transmission. Usually the DC subcarrier and some carriers at the edge of the transmission band are not used due to technical difficulties and guard band purposes in the spectrum. Clipping introduces nonlinear distortions in the entire baseband, so the originally unused subcarriers will contain components introduced by clipping. This also negatively affects the spectral behavior of the transmission signal, that is, leakage will appear. These components have to be suppressed. Digital filtering is not sufficient to suppress the clipping components on the unused subcarriers and analog filtering introduces modulation errors. Instead of filtering, the clipped transmit signal is demodulated again. The modulation values for each symbols of the used subcarriers are selected and the unused subcarriers are set to zero, and repeated modulation is performed similar as described in [30]. The described modification of the transmitter for OFDM and SMT systems can be seen in Figure 10. In the receiver the BNC detector for the OFDM system remains the same, as the compensation of the clipping noise is performed in the frequency domain. On the other hand, the BNC receiver for the SMT scheme has to be modified. The same scheme as presented in Figure 10 must be implemented in the feedback loop to reconstruct the clipping noise in the time domain.

The results of clipping and resetting the unmodulated subcarriers to zero for OFDM and SMT can be seen in Figures 11 and 12. In Figure 11 a spectrum regrowth can be 


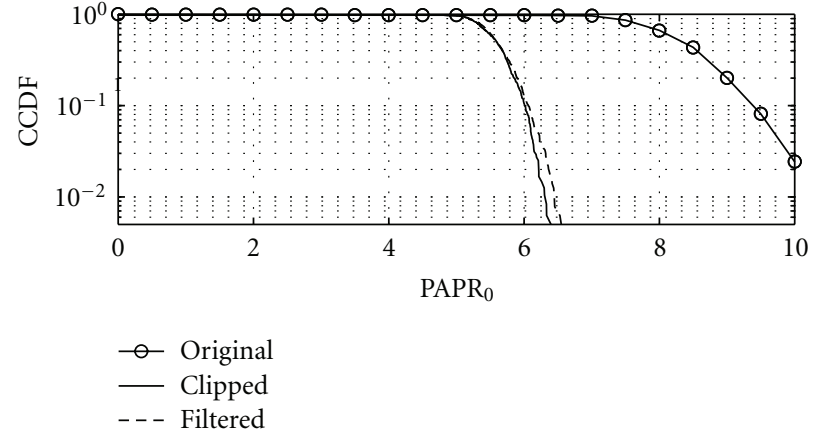

(a) OFDM

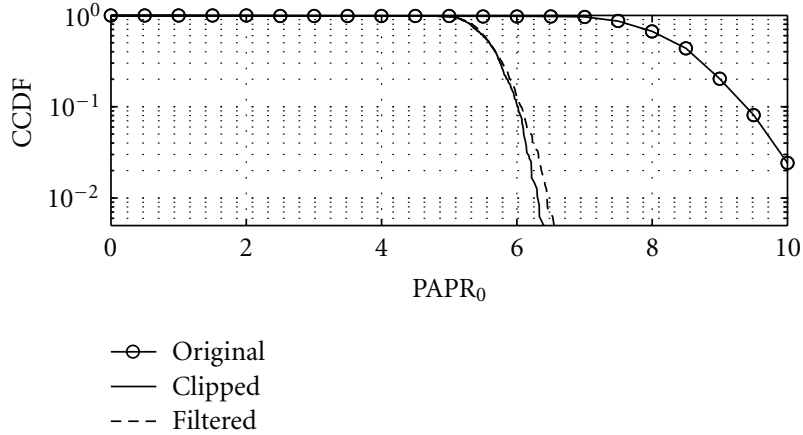

(b) SMT

FIGURE 11: CCDF of the PAPR values of the transmitted signal with clipping and additional signal processing for OFDM (a) and SMT (b).

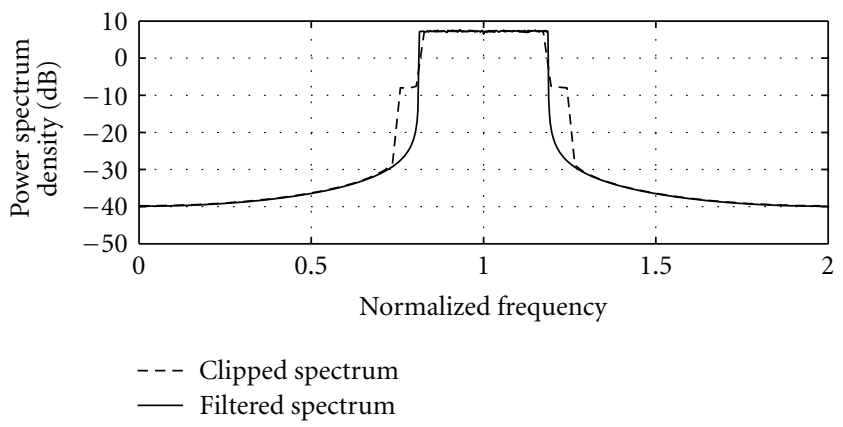

(a) OFDM

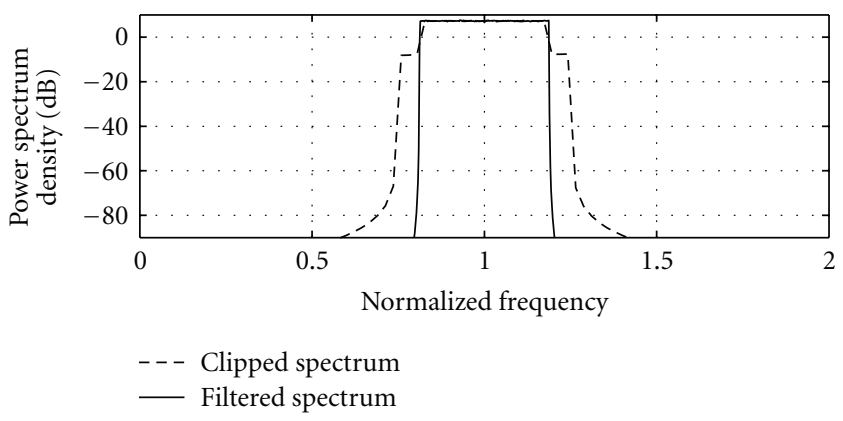

(b) SMT

FIGURE 12: Power spectrum density function of the transmitted signal with clipping and additional signal processing for OFDM (a) and SMT (b).

observed for the PAPR of both systems as a result of the filtering. The regrowth of PAPR is strongly dependent on number of unused subcarriers. If more unmodulated subcarriers are applied, more nonlinear distortion will be removed from the signal leading to the regrowth of the peaks. In Figure 12 on the spectral characteristics the effects of clipping can be well observed as the nonlinear products appear in the baseband causing a large side lobe. This leakage disappears with the filtering of the unused subcarriers. As a positive sideeffect of the filtering the power of the clipping noise will also be reduced, so the receiver will operate with a reduced $P_{d}$.

\section{Simulation Results}

Table 1 shows the summary of the simulation parameters for the two modulation schemes.

The binary data are encoded with a code rate of $1 / 2$, using a 4-state recursive systematic convolutional encoder with polynomials $(1,5 / 7)_{8}$ in octal notation. The interleaved bits are mapped according to a 16-QAM constellation with Gray mapping. The clipping level (CR) is set to $1 \mathrm{~dB}$. For the prototype filter of the SMT system the coefficients presented in [31] are applied.
TABLE 1: Simulation parameters for SMT and OFDM system.

\begin{tabular}{lcc}
\hline Parameter & SMT & \multicolumn{2}{c}{ OFDM } \\
Bandwidth & \multicolumn{2}{c}{$8 \mathrm{MHz}$} \\
Cyclic prefix $(P)$ length & 0 & \multicolumn{2}{c}{128} \\
Available subcarriers/subbands $(N)$ & \multicolumn{2}{c}{1024} \\
Modulated subcarriers/subbands $\left(N_{c}\right)$ & \multicolumn{2}{c}{768} \\
Overlapping factor $(K)$ & 4 & \multicolumn{2}{c}{1} \\
Mapping $(M)$ & \multicolumn{2}{c}{$4(16-\mathrm{QAM})$} \\
Clipping ratio & \multicolumn{2}{c}{$1 \mathrm{~dB}$} \\
\hline
\end{tabular}

To obtain comparable bit error rates, the SNR normalized to one bit energy is defined. The noise power of the AWGN channel is calculated according to the following definition:

$$
\begin{aligned}
\mathrm{SNR}_{\mathrm{dB}} & =10 \log _{10}\left(\frac{P_{b}}{N_{0}}\right) \\
& =10 \log _{10}\left(\frac{P_{\text {out }}(N+P)}{N_{0} M R N_{c}}\right),
\end{aligned}
$$

where $P_{b}$ is the bit power, $N$ is the number of the subcarriers/subbands available, and $N_{c}$ is the number of subcarriers/subbands used. $P$ is the length of the $\mathrm{CP}, M$ is the number 
TABLe 2: Excess delay and relative amplitude for IEEE 802.22 B and C channel profiles.

\begin{tabular}{lcccccc}
\hline Profile B & Path 1 & Path 2 & Path 3 & Path 4 & Path 5 & Path 6 \\
\hline Excess d. & $-3 \mu \mathrm{s}$ & $0 \mu \mathrm{s}$ & $2 \mu \mathrm{s}$ & $4 \mu \mathrm{s}$ & $7 \mu \mathrm{s}$ & $11 \mu \mathrm{s}$ \\
Rel. amp. & $-6 \mathrm{~dB}$ & $0 \mathrm{~dB}$ & $-7 \mathrm{~dB}$ & $-22 \mathrm{~dB}$ & $-16 \mathrm{~dB}$ & $-20 \mathrm{~dB}$ \\
\hline Profile C & Path 1 & Path 2 & Path 3 & Path 4 & Path 5 & Path 6 \\
\hline Excess d. & $-2 \mu \mathrm{s}$ & $0 \mu \mathrm{s}$ & $5 \mu \mathrm{s}$ & $16 \mu \mathrm{s}$ & $24 \mu \mathrm{s}$ & $33 \mu \mathrm{s}$ \\
Rel. amp. & $-9 \mathrm{~dB}$ & $0 \mathrm{~dB}$ & $-19 \mathrm{~dB}$ & $-14 \mathrm{~dB}$ & $-24 \mathrm{~dB}$ & $-16 \mathrm{~dB}$ \\
\hline
\end{tabular}

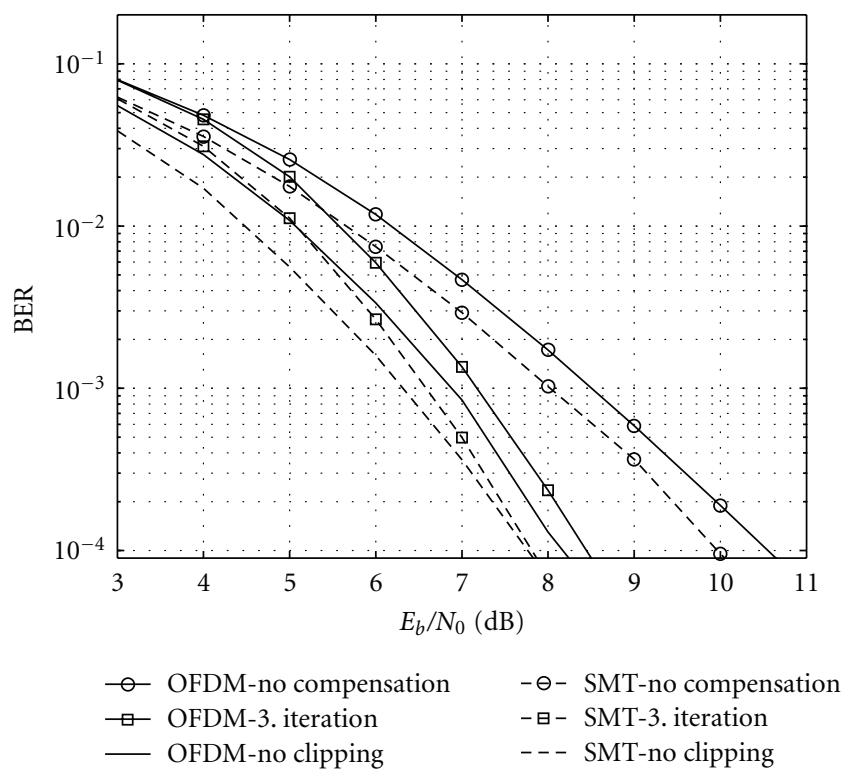

Figure 13: Bit error rates of the BNC receiver for OFDM and SMT signaling over AWGN channel.

of bits transmitted by one subcarrier/subband, and $R$ is the coding rate. Assuming a normalized symbol duration $T$, the energy of a single bit can be expressed as $E_{b}=P_{b} T=P_{b}$. The parameters of the applied IEEE 802.22 channel profiles $\mathrm{B}$ and $\mathrm{C}$ can be found in Table 2 [32]. For decoding of the received bits BJCR decoder was suggested, but due to arithmetic overflow issues the log-map decoder [33] is used.

The simulated BERs over AWGN channel can be seen in Figure 13. Due to the absence of CP, SMT outperforms OFDM. It can be observed for both techniques that clipping severely degrades the overall system performance if it is not compensated, that is, no feedback loop is active. With iterative compensation the system performance can be improved, if the SNR acceded a limit the BER results approach the performance of the case without clipping.

The BER simulations for Channel B can be seen in Figure 14. For OFDM the CP is longer than the maximal channel delay, therefore ISI is not affecting the OFDM system, and the effects of clipping can be compensated. For SMT the effect of the ISI does not severely degrade the system performance, it still outperforms OFDM. For both techniques the effect of clipping can be compensated and the BER after the third iteration approaches the results where no clipping was applied.

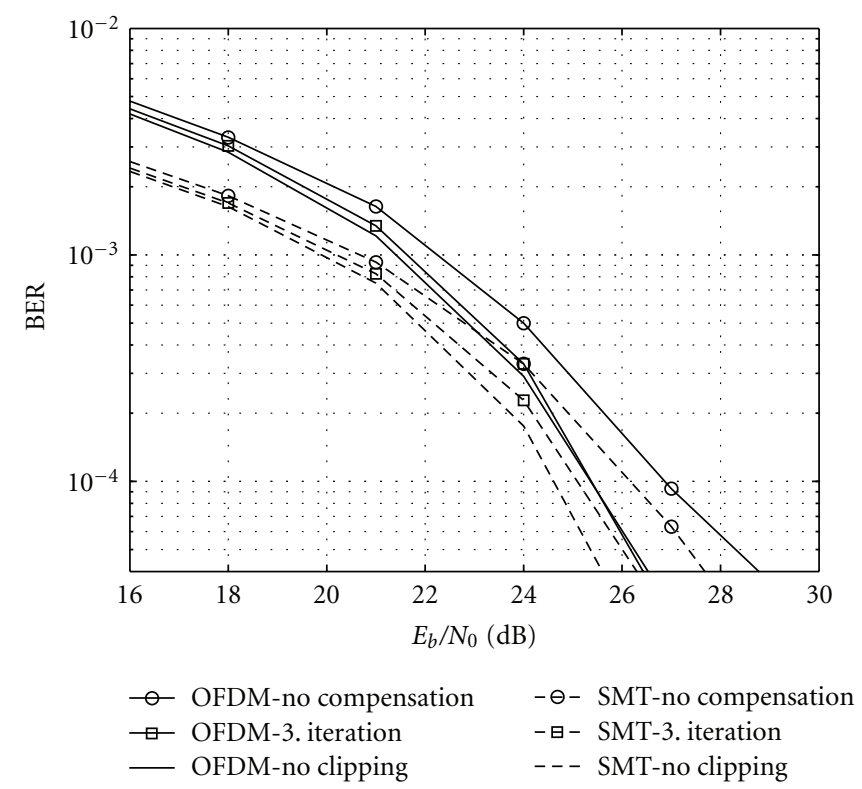

FIGURE 14: Bit error rates of the BNC receiver for OFDM and SMT signaling over channel B.

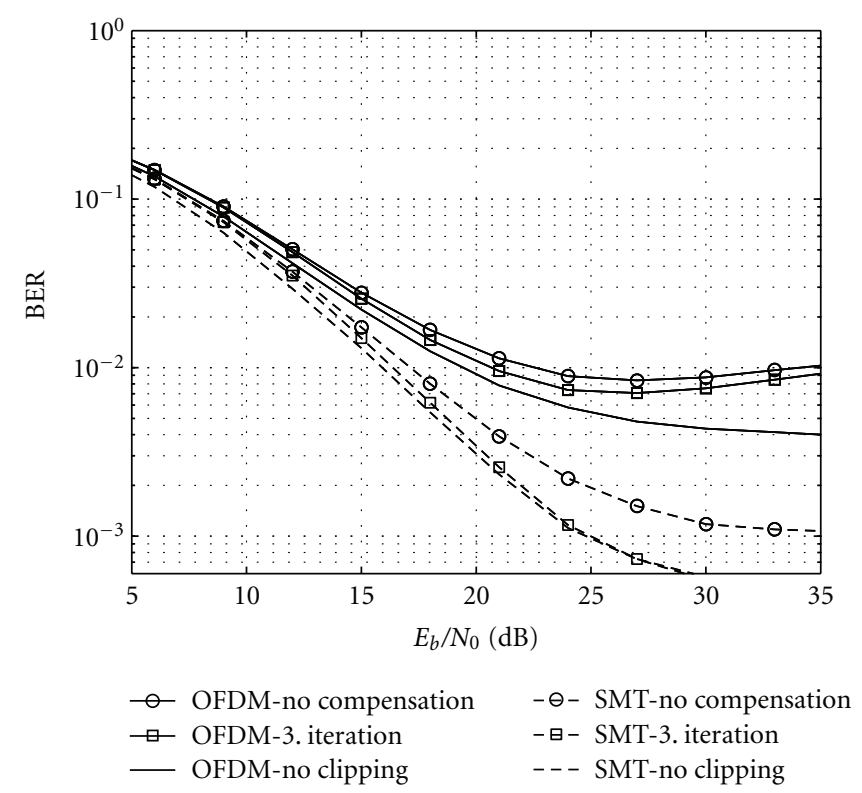

FIGURE 15: Bit error rates of the BNC receiver for OFDM and SMT signaling over channel C.

The BER simulations for Channel $\mathrm{C}$ are shown in Figure 15. In this scenario the CP of OFDM is shorter than the channel impulse response so an error floor caused by the residual ISI can be observed. The presence of ISI results in an error floor also for FBMC systems but at a much lower SMT.

\section{Conclusion}

In this paper a modified BNC structure suitable for clipped SMT signal processing was presented. Based on the EXIT 
chart, it was shown that the proposed iterative scheme is convergent. It was also described how the clipping technique can be applied in real-life systems for both OFDM and SMT modulation. Finally, the performance of the BNC SMT receiver was verified and compared to OFDM based on BER simulations over AWGN and Rayleigh channels. For both systems the clipping compensation can be performed and the performance without clipping can be approached.

\section{Acknowledgment}

The research leading to these results was derived from the European Community's Seventh Framework Programme (FP7) under Grant Agreement no. 248454 (QoSMOS).

\section{References}

[1] A. R. S. Bahai, B. R. Saltzberg, and M. Ergen, Multi Carrier Digital Communications: Theory and Applications of OFDM, Springer, 2004.

[2] J. Gazda, P. Drotár, D. Kocur, P. Galajda, and R. Blicha, "Joint evaluation of nonlinear distortion effects and signal metrics in OFDM based transmission systems," Acta Electrotechnica et Informatica, vol. 9, no. 4, pp. 55-60, 2009.

[3] S. H. Han and J. H. Lee, "An overview of peak-to-average power ratio reduction techniques for multicarrier transmission," IEEE Wireless Communications, vol. 12, no. 2, pp. 56-65, 2005.

[4] P. Foomooljareon and W. Fernando, "PAPR reduction in OFDM systems," Thammasat International Journal of Science and Technology, vol. 7, no. 3, 2002.

[5] X. Li and L. J. Cimini, "Effects of clipping and filtering on the performance of OFDM," IEEE Communications Letters, vol. 2, no. 5, pp. 131-133, 1998.

[6] A. E. Jones, T. A. Wilkinson, and S. K. Barton, "Block coding scheme for reduction of peak to mean envelope power ratio of multicarrier transmission schemes," Electronics Letters, vol. 30, no. 25, pp. 2098-2099, 1994.

[7] A. D. S. Jayalath and C. Tellambura, "The use of interleaving to reduce the peak to average power ratio of an OFDM signal," in Proceedings of the IEEE Global Telecommunication Conference (GLOBECOM '00), vol. 1, pp. 82-86, 2000.

[8] S. H. Müller and J. B. Huber, "OFDM with reduced peakto-average power ratio by optimum combination of partial transmit sequences," Electronics Letters, vol. 33, no. 5, pp. 368369, 1997.

[9] A. Mobasher and A. K. Khandani, "Integer-based constellation-shaping method for PAPR reduction in OFDM systems," IEEE Transactions on Communications, vol. 54, no. 1, pp. 119127, 2006.

[10] S.-E. Park, Y. Sung-Ryul, J. Y. Kim, D. S. Park, and P. Y. Joo, "Tone reservation method for PAPR reduction scheme," Tech. Rep. IEEE 802.16e Task Group, IEEE 802.16e-03n60, 2003.

[11] S. H. Han, J. M. Cioffi, and J. H. Lee, "Tone injection with hexagonal constellation for peak-to-average power ratio reduction in OFDM," IEEE Communications Letters, vol. 10, no. 9, pp. 646-648, 2006.

[12] B. S. Krongold and D. L. Jones, "PAR reduction in OFDM via active constellation extension," IEEE Transactions on Broadcasting, vol. 49, no. 3, pp. 258-268, 2003.
[13] B. Farhang-Boroujeny and C. H. Yuen, "Cosine modulated and offset QAM filter bank multicarrier techniques: a continuous-time prospect," Eurasip Journal on Advances in Signal Processing, vol. 2010, Article ID 165654, 2010.

[14] F. Schaich, "Filterbank based multi carrier transmission (FBMC)—evolving OFDM: FBMC in the context of WiMAX," in Proceedings of the European Wireless Conference (EW '10), pp. 1051-1058, April 2010.

[15] P. Siohan, C. Siclet, and N. Lacaille, "Analysis and design of OFDM/OQAM systems based on filterbank theory," IEEE Transactions on Signal Processing, vol. 50, no. 5, pp. 1170-1183, 2002.

[16] M. Colas, G. Gelle, and D. Declercq, "Analysis of iterative receivers for clipped COFDM signaling based on soft TurboDAR," in Proceedings of the 1st International Symposium on Wireless Communication Systems (ISWCS '04), pp. 110-114, September 2004.

[17] H. Chen and A. Haimovich, "An iterative method to restore the performance of clipped and filtered OFDM signals," in Proceedings of the International Conference on Communications (ICC '03), pp. 3438-3442, May 2003.

[18] R. Djardin, M. Colas, and G. Gelle, "Comparison of iterative receivers mitigating the clipping noise of OFDM based system," in Proceedings of the European Wireless Conference, 2007.

[19] Z. Kollár, M. Grossmann, and R. Thomä, "Convergence analysis of BNC turbo detection for clipped OFDM signalling," in Proceedings of the 13th International OFDM-Workshop (InOWo'08), pp. 241-245, Hamburg, Germany, 2008.

[20] D. S. Waldhauser and J. A. Nossek, "Multicarrier systems and filter banks," Advances in Radio Science, vol. 4, pp. 165-169, 2006.

[21] Z. Kollár, G. Péceli, and P. Horváth, "Iterative decision feedback equalization for FBMC systems," in Proceedings of the 1st International Conference on Advances in Cognitive Radio (COCORA '11), Budapest, Hungary, 2011.

[22] T. Ihalainen, T. H. Stitz, and M. Renfors, "Efficient per-carrier channel equalizer for filter bank based multicarrier systems," in Proceedings of the IEEE International Symposium on Circuits and Systems (ISCAS '05), pp. 3175-3178, jpn, May 2005.

[23] Z. Kollár and P. Horváth, "Modulation schemes for cognitive radio in white spaces," Radioengineering, vol. 19, no. 4, pp. 511-517, 2010.

[24] L. Baltar, D. S. Waldhauser, and J. A. Nossek, "Out-of-band radiation in multicarrier systems: a comparison," Multi-Carrier Spread Spectrum, vol. 1, pp. 107-116, 2007.

[25] H. E. Rowe, "Memoryless non-linearities with gaussian inputs: elementary results," The Bell System Technical Journal, vol. 61, no. 7, pp. 1519-1525, 1982.

[26] S. Ten Brink, "Designing iterative decoding schemes with the extrinsic information transfer chart," AEU-Archiv fur Elektronik und Ubertragungstechnik, vol. 54, no. 6, pp. 389-398, 2000.

[27] S. ten Brink, J. Speidel, and R. H. Yan, "Iterative demapping and decoding for multilevel modulation," in Proceedings of the Global Telecommunications Conference, (GLOBECOM '98), pp. 579-584, November 1998.

[28] L. R. Bahl, J. Cocke, F. Jelinek, and J. Raviv, "Optimal decoding of linear codes for minimizing symbol error rate," IEEE Transactions on Information Theory, vol. IT-20, no. 2, pp. 284287, 1974.

[29] "Phydyas project: documents D2.1 and D3.1," 2008, http:// www.ict-phydyas.org/.

[30] P. Sharma, S. Verma, and A. Basu, "Modified clipping and filtering technique for peak-to-average power ratio reduction 
of OFDM signals used in WLAN," International Journal of Engineering Science and Technology, vol. 2, no. 10, pp. 5337$5343,2010$.

[31] M. Bellanger, "Physical layer for future broadband radio systems," in Proceedings of the IEEE Radio and Wireless Symposium (RWW'10), pp. 436-439, January 2010.

[32] E. Sofer and G. Chouinard, "WRAN channel modeling," IEEE 802.22-05/0055r7, 2005.

[33] G. Bauch, Turbo-Entzerrung, und Sendeantennen Diversity mit Space-Time-Codes im Mobilfunk, VDI Verlag GmbH, Düsseldorf, Germany, 2001. 

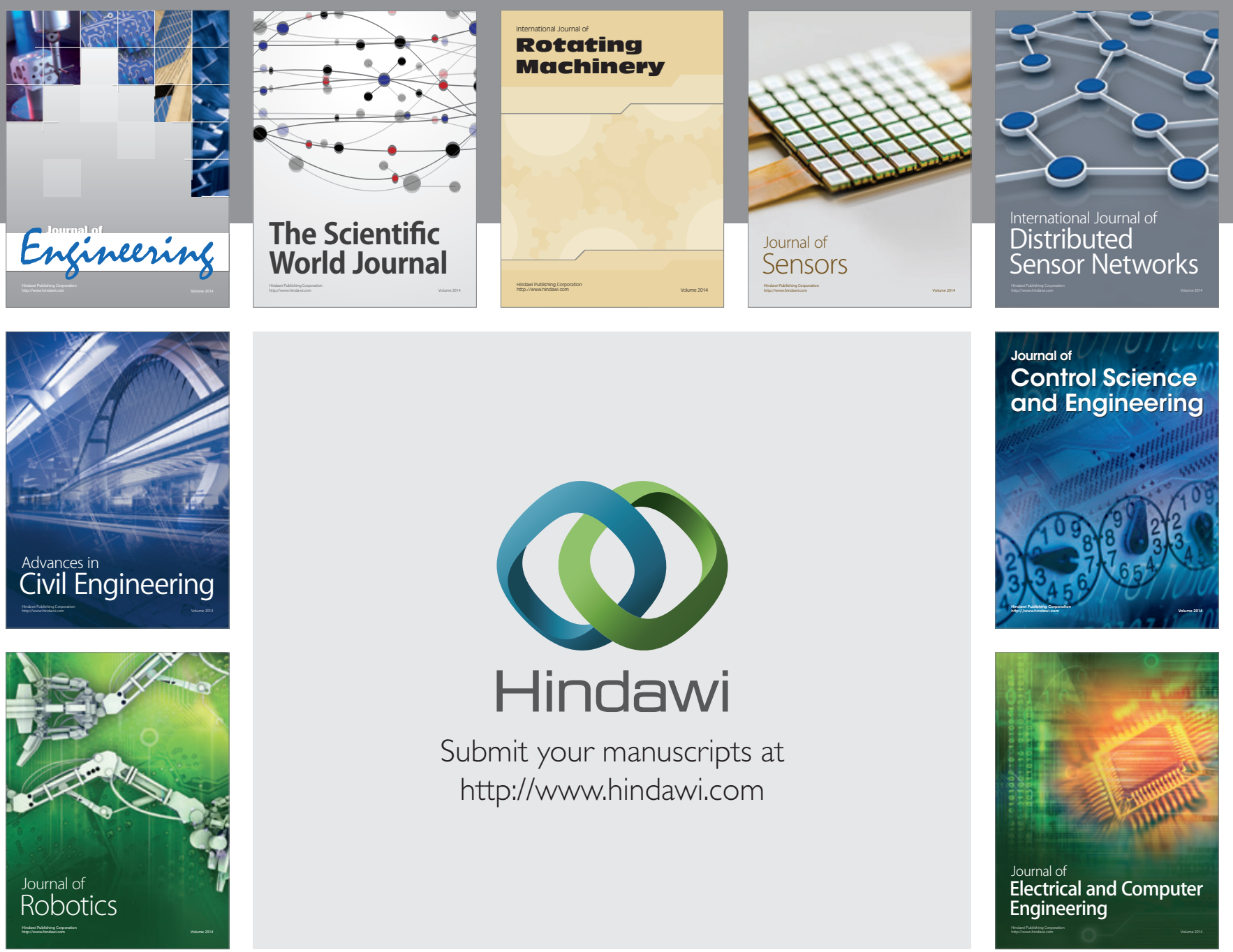

Submit your manuscripts at

http://www.hindawi.com
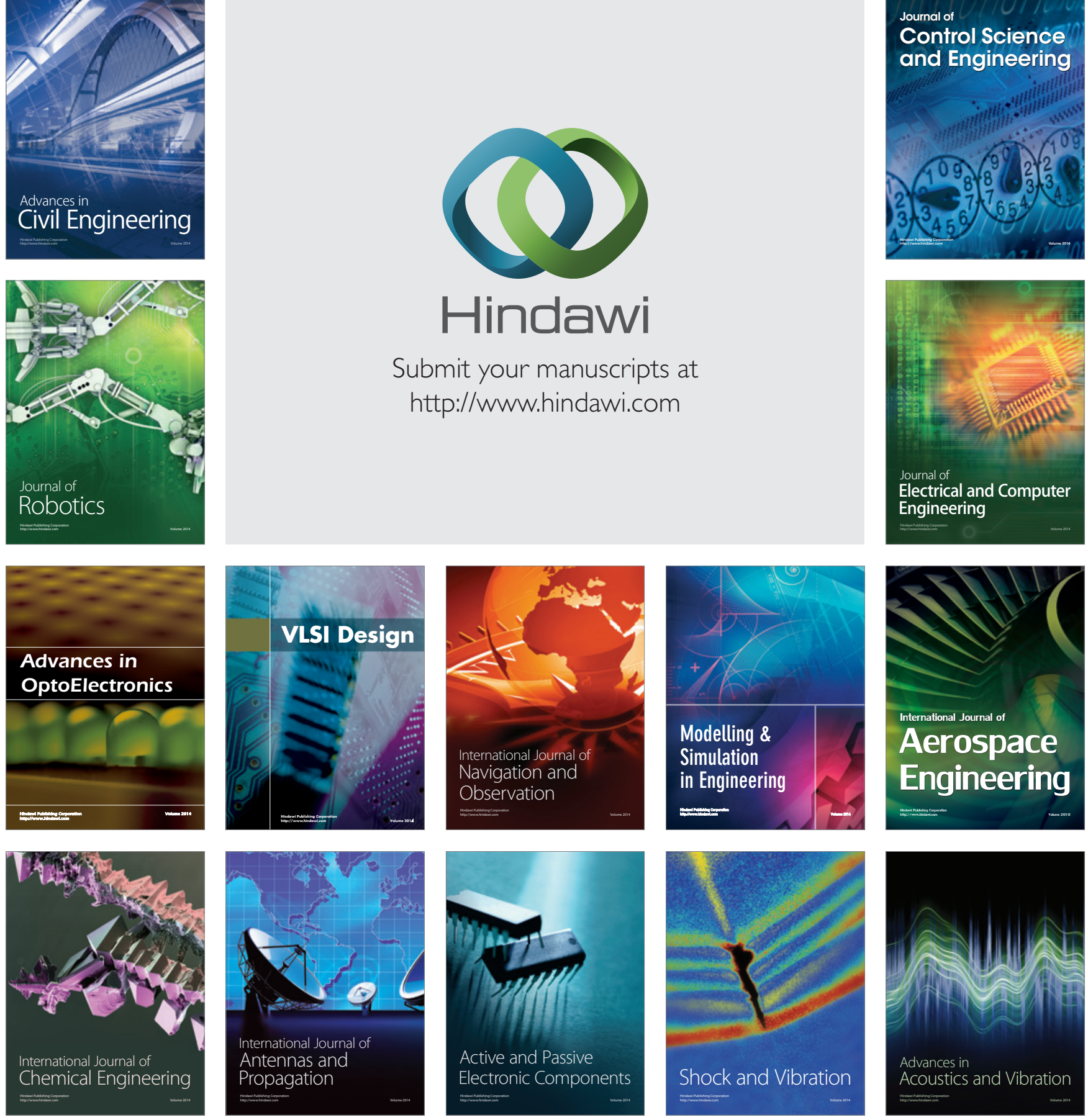